\title{
CAN EVOLUTION IN CLOSE BINARIES ACCOUNT FOR THE BLUE STRAGGLERS IN M67?
}

\author{
Alejandra A. E. Milone \\ Harvard-Smithsonian Center for Astrophysics and \\ Córdoba Observatory, National University of Córdoba \\ Laprida 854, 5000 Córdoba, Argentina \\ David W. Latham \\ Harvard-Smithsonian Center for Astrophysics \\ 60 Garden Street, Cambridge, Massachusetts 02138, U.S.A. \\ Robert D. Mathieu \\ Department of Astronomy, University of Wisconsin \\ 475 Charter Street, Madison, Wisconsin 53706, U.S.A. \\ Jon A. Morse \\ Department of Physics and Astronomy, University of North Carolina \\ CB \#3255, Phillips Hall, Chapel Hill, NC 27599-3255, U.S.A. \\ Robert J. Davis \\ Harvard-Smithsonian Center for Astrophysics \\ 60 Garden Street, Cambridge, Massachusetts 02138, U.S.A.
}

For the past 9 years we have been monitoring the radial velocities of 13 blue stragglers in the old open cluster M67. For the 9 blue stragglers with rotational velocities no larger than about $100 \mathrm{~km} \mathrm{~s}^{-1}$ we have used the CfA digital speedometers to measure more than $\mathbf{5 0 0}$ radial velocities. To get reliable velocity correlations we use synthetic rotating templates computed from a grid of Kurucz model atmospheres. Four of the blue stragglers rotate too rapidly to allow successful velocity correlations with the CfA instruments. For three of these we have used a CCD spectrograph at Kitt Peak and similar reduction procedures (Morse et al. 1991.

In Table 1 we summarize the status of the radial-velocity measurements as of mid 1991. The identifications are taken from Sanders (1977) and Fagerholm (1906). Positions and photometry may be found in Girard et al. (1989). The effective temperatures and rotational velocities were chosen to give the best correlations with synthetic spectra calculated from Kurucz model atmospheres. Note that the probability of membership in M67 is very high for all these blue stragglers, based on their proper motions (Girard et al. 1989), and that the mean radial velocities are all consistent with membership as well.

For stars with constant velocities, the rms deviations of the CfA velocities should be 1 or $2 \mathrm{~km} \mathrm{~s}^{-1}$, depending on the rotation. Thus, it appears that at least 5 of the 9 blue stragglers measured with the CfA instruments have variable velocities, with the possibility that as many as 7 may be variable. Are most of the blue stragglers binaries, and has mass transfer been involved in making them into blue stragglers (McCrea 1964, Collier \& Jenkins 1984)? One of the blue stragglers, F190, has a short period. An orbital 
Table 1. Mean radial velocities for thirteen blue stragglers in M67

\begin{tabular}{|c|c|c|c|c|c|c|c|c|}
\hline \multicolumn{2}{|c|}{ Star } & \multirow{2}{*}{$\begin{array}{r}\begin{array}{r}T_{\text {eff }} \\
(\mathrm{K})\end{array} \\
6500\end{array}$} & \multirow{2}{*}{$\begin{array}{c}\begin{array}{c}\text { Rotational } \\
\text { Velocity } \\
\left(\mathrm{km} \mathrm{s}^{-1}\right)\end{array} \\
0\end{array}$} & \multirow{2}{*}{$\begin{array}{c}P(\mu, r) \\
(\%)\end{array}$} & \multirow{2}{*}{$\frac{N_{\text {obs }}}{70}$} & \multicolumn{2}{|c|}{ Mean Radial } & \multirow{2}{*}{$\frac{\begin{array}{c}\mathrm{rms} \\
\left(\mathrm{km} \mathrm{s}^{-1}\right)\end{array}}{1.75}$} \\
\hline S1082 & F131 & & & & & 33.37 & $\mathrm{C}$ & \\
\hline S975 & F90 & 6500 & 20 & $?$ & 35 & 33.61 & C & 5.02 \\
\hline S997 & F124 & 6500 & 20 & 99 & 28 & 35.51 & C & 5.09 \\
\hline S1195 & F207 & 6500 & 60 & 98 & 29 & 30.30 & C & 7.54 \\
\hline S1263 & F185 & 7000 & 20 & 100 & 38 & 32.06 & C & 0.99 \\
\hline S1280 & F184 & 7000 & $>100$ & 100 & & & & \\
\hline S1267 & F238 & 7500 & 40 & 99 & 25 & 35.08 & C & 2.88 \\
\hline S752 & F55 & 7500 & 80 & 99 & 45 & 35.48 & C & 5.14 \\
\hline S1284 & F190 & 7500 & 80 & 99 & 62 & 33.70 & C & 11.33 \\
\hline S968 & F153 & 8000 & 0 & 99 & 72 & 32.50 & C & 0.83 \\
\hline S1434 & F280 & 9000 & 125 & 95 & 3 & 33.04 & K & 3.81 \\
\hline S1066 & F156 & 9250 & 80 & 100 & 3 & 34.31 & $\mathbf{K}$ & 1.67 \\
\hline S977 & F81 & 14000 & 125 & 99 & 7 & 33.42 & $\mathrm{~K}$ & 2.50 \\
\hline
\end{tabular}

solution for this star is reported by Milone \& Latham (1992), who argue that it is in the final stages of the mass transfer that made it into a blue straggler.

The velocity variations of S975, S997, and S1195 look like they are due to orbital motion with periods longer than 1000 days and semiamplitudes under $10 \mathrm{~km} \mathrm{~s}^{-1}$. It is not impossible that these three systems are binaries in the final slow stages of mass transfer. The original primary has by now donated a large fraction of its initial mass to the original secondary, which we now see as the blue straggler. The orbital period can easily be thousands of days for this stage of mass transfer, and the lifetime in this stage can be similar to the main-sequence lifetime of the original primary (Paczyński 1971). All three of these blue stragglers lie off of the Zero Age Main Sequence. Normal single stars would not spend very long in this region of the color-magnitude diagram. Perhaps the progress of the blue stragglers across the colormagnitude diagram has been slowed by the ongoing mass transfer.

An alternative mechanism for forming blue stragglers may involve binary-binary interactions ( $c f$. Leonard 1989, Leonard \& Fahlman 1991). In this scenario the blue straggler would result from a merger of two of the stars, the third star would be the distant companion corresponding to the long periods that we observe, and the fourth would be ejected from the system by the encounter.

We thank Bob Kurucz for his help in calculating the grid of rotating synthetic spectra from his model atmospheres, and Guillermo Torres for his help with the velocity correlations.

\section{REFERENCES}

Collier, A. C., \& Jenkins, C. R. 1984, MNRAS, 211, 391

Fagerholm, E. 1906, Inaugural Dissertation, Uppsala

Girard, T. M., Grundy, W. M., Lopez, C. E., \& van Altena, W. F. 1989, AJ, 98, 227

Leonard, P. J. T. 1989, AJ, 98, 217

Leonard, P. J. T., \& Fahlman, G. G. 1991, AJ, in press

McCrea, W. H. 1964, MNRAS, 128, 147

Milone, A. A. E., \& Latham, D. W. 1992, this volume

Morse, J. A., Mathieu, R. D., and Levine, S. E. 1991, AJ, 101, 1495

Paczyński, B. 1971, ARA\&A, 9, 183

Sanders, W. L. 1977, A\&AS, 27, 89 